\title{
USO DE LAS NUEVAS TECNOLOGÍAS POR LAS PERSONAS MAYORES EN UNA LOCALIDAD RURAL
}

\author{
Mónica Matellanes-Lazo1: Universidad Europea Miguel de Cervantes de \\ Valladolid, España. \\ mmatellanes@uemc.es
}

\section{RESUMEN}

Con este artículo, se pretende realizar una visión de las posibilidades que ofrecen las Nuevas Tecnologías de la Información y la Comunicación para el aprendizaje, concretamente para su uso por las personas mayores, entendiendo que nos encontramos ante unos medios y canales de uso y disfrute exclusivo por los jóvenes, como se viene pensando desde diferentes instancias. Máxime, si tenemos en cuenta que cada vez nos encontramos con un número mayor de personas mayores que deciden dar respuesta a interrogantes o ambiciones, que por algún motivo, no han podido ser resueltas en épocas anteriores de su vida.

Los últimos avances en Tecnología de la Información y Comunicación ofrecen a los casi 8 millones de españoles que superan los 65 años, soluciones que les ayudan a permanecer activos socialmente.

Para ello se ha realizado un breve cuestionario descriptivo para la obtención de los objetivos marcados. Los resultados aportan un bajo y ligero uso y crecimiento de Internet y de las nuevas tecnologías en una población rural llamada Cabezón de Pisuerga de la provincia de Valladolid.

PALABRAS CLAVE: Nuevas tecnologías - Mayores - Comunicación - Internet Envejecimiento - Interacción.

\footnotetext{
${ }^{1}$ Autor correspondiente:

Mónica Matellanes-Lazo: Profesora Doctora Agregada de la Universidad Europea Miguel de Cervantes de Valladolid (España). Experta en Marketing y Comunicación Corporativa. Coordinadora Académica del Grado de Publicidad y RR.PP: Correo: mmatellanes@uemc.es
} 


\title{
NEW TECHNOLOGIES CUSTOMS BY ELDERLY PEOPLE IN A COUNTRYSIDE
}

\begin{abstract}
With this article, we seek to carry out a vision of the possibilities that you/they offer the New Technologies of the Information and the Communication for the learning, concretely for their use for the grown-ups, understanding that we are not before some use means and exclusive enjoyment for the youths, like one comes thinking from different instances. Maximum, if we keep in mind that every time we meet with a number bigger than grown-ups that decide to give answer to queries or ambitions that for some reason, they have not been able to be solved in times previous of their life.

With a short questionnaire made to the old people in a countryside of Valladolid, we obtained a viewpoint of Internet customs by this kind of elderly people in Cabezón de Pisuerga countryside.
\end{abstract}

KEY WORDS: New Technologies - Elderly people- Communication - Internet Ageing - Interaction.

\section{INTRODUCCIÓN}

Posiblemente no podamos olvidar que nunca las tecnologías se habían multiplicado como hasta la fecha, de manera que nos encontramos ya no sólo con las que podríamos considerar como tecnologías tradicionales, sino con las denominadas nuevas tecnologías. Asumiendo de entrada que los conceptos "tradicionales" y "nuevo" deben de ser tomados con cierta cautela, la realidad es que frente a las tecnologías industriales y tradicionales de la revolución industrial o de primera y segunda ola, utilizando la terminología del célebre visionario contemporáneo Alvin Toffler, en la actualidad existe una diversidad y multitud de tecnologías que van desde la microelectrónica, a las telecomunicaciones, pasando por las biotecnologías, sin olvidar las tecnologías renovables; como no había ocurrido anteriormente.

Todos estaremos de acuerdo con la afirmación que la sociedad ha pasado por diferentes revoluciones tecnológicas, que por lo general se encuadran en tres: agrícola, industrial y de la información. Si la primera vino marcada, por la utilización de la fuerza de los animales, la rotación de los cultivos y la automatización de la agricultura y la selección de las semillas, y la segunda, por el desarrollo de las primeras industrias textiles y de acero, y la aparición de la electricidad (Duby, 1991). 
La actual de la información, adopta como elemento básico de desarrollo tecnológico la información, poniendo el énfasis no en los productos sino en los procesos, y desarrollándose no de forma aislada sino en interconexión y refuerzo mutuo. Sociedad en red, como la denominan algunos, que proviene de la revolución de las tecnologías de la información, la reestructuración del capitalismo y la desaparición del estatismo.

Sociedad tecnológica, de la información o en red, que viene configurada por una serie de características que sin la pretensión de acotarlas podemos especificarlas en las siguientes:

- Globalización de las actividades económicas.

- Incremento del consumo y producción masiva de los bienes de consumo.

- Sustitución de los sistemas de producción mecánicas, por otros de carácter electrónicos y automáticos.

- Modificación de las relaciones de producción, tanto social como desde una posición técnica.

- Flexibilización del trabajo e inestabilidad laboral.

- Aparición de nuevos sectores laborales, como el dedicado a la información y de nuevas modalidades laborales como el teletrabajo.

- Globalización de los medios de comunicación de masas tradicionales, e interconexión de las tecnologías tanto tradicionales como novedosas, de manera que permitan romper las barreras espacio-temporales y el alcance de grandes distancias.

- La transformación de la política y de los partidos políticos, estableciéndose nuevos mecanismos para la lucha por el poder.

- Tendencia a la americanización de la sociedad.

Las repercusiones de la sociedad de la información producto de la utilización de las nuevas tecnologías de la información y comunicación, supera los límites de la cantidad de información que puede ser puesta a disposición de las personas, la velocidad con que puede ser transferida, o la eficacia con que puede llegar a su destino, para alcanzar a todos los sectores sociales, teniendo repercusiones directa en el mundo laboral con la aparición de nuevos sectores gremiales, la potenciación de nuevas profesiones, la desaparición de otras, la transformación de determinadas actividades laborales adecuándolas a los tiempos, y lo que puede ser más significativo la posibilidad de que el sujeto desarrolle a lo largo de su vida diferentes actividades profesionales (González, 1996); sin olvidarnos de sus repercusiones en los diferentes estadios de nuestra vida.

Como estamos viendo, la utilización de las nuevas tecnologías en esta sociedad de la información no será sólo "un asunto tecnológico; es básicamente un gran desafío 
político, cultural, económico y social" (Torres, 1994: 91). Estamos ante una verdadera revolución en todos los contextos del ser humano, desde lo político, a lo económico, a lo social y lo cultural.

Esta sociedad de la información se ha visto impulsada por diferentes medios, de ellos los fundamentales son la informática, las telecomunicaciones y las redes de comunicación. En cuanto a la informática, su impacto ha sido de tal forma que podría ser comparada con el descubrimiento de la rueda o el fuego por el ser humano; ahora bien, su significación en la nueva cultura, no ha sido exclusivamente como instrumento que nos permite escribir, calcular, simular, guardar, tratar información y comunicarnos con los demás, sino lo que es más importante nos está ofreciendo nuevos modelos de mente con la simulación donde proyectar nuestras ideas y fantasías (Turkle, 1997: 15).

Claramente determinadas por la aparición de la informática, nos encontramos con las telecomunicaciones y las denominadas autopistas de la información que provienen de la interacción de diferentes tecnologías de la información y comunicación con, y gracias a, la informática. Al mismo tiempo no debemos de olvidarnos de las mutaciones que se ofrecen las mismas, como los multimedia.

Es importante también percibir que las redes de comunicación no están exclusivamente formadas por los medios tecnológicos e instrumentales, sino también, y puede ser lo importante, por todos los contenidos y mensajes, que son generados por los usuarios de las redes, como por aquellos que son transportados, directa o elaboradamente, por el usuario desde la red. Las redes más que ser redes de intercambio de información, son redes de interacción entre personas.

La influencia de estas nuevas tecnologías de la información y comunicación van a tener una repercusión significativa en la sociedad del futuro, proponiendo cambios que van desde las formas de acercarnos y generar el conocimiento, hasta el tipo de interacción que establecemos entre las personas.

Para Gates (1995) uno de los "gurús" de la sociedad de la información, la influencia de estas nuevas tecnologías va tener diferentes repercusiones en la sociedad, como son: un acceso a la información ilimitada por parte de todos y en cualquier momento, una individualización de la enseñanza por parte de los docentes, mediante la utilización de multimedia y programas de autor, alargar y divulgar el conocimiento y la investigación entre los docentes y los investigadores, la posibilidad que los docentes van a tener para superar la metodología expositiva centrándonos en la resolución de los problemas, una mayor participación-colaboración del medio escolar como una comunidad educativa envolvente.

Ramonet (1997), nos habla con toda claridad que uno de los peligros que nos encontramos en la actualidad, es que las nuevas tecnologías de la información y comunicación lleguen a separar al mundo en dos grandes categorías: "infopobres" e 
"inforicos", con un fuerte sometimiento y supeditación de los primeros a los segundos.

Posiblemente sea ahora el momento de preguntarnos, qué es lo que vamos entender por nuevas tecnologías de la información y comunicación. Y en este caso podemos decir que son aquellas que giran de manera interactiva en torno a las telecomunicaciones, la informática y los audiovisuales y su hibridación como son los multimedia. De todas formas, no podemos olvidarnos que el paradigma actual de las nuevas tecnologías son las redes informáticas, que nos permiten en la interacción de los ordenadores ampliar la potencia y funcionalidad que tienen de forma individual, permitiéndonos no solo procesar información almacenada en soportes físicos, sino también acceder a recursos y servicios prestados por ordenadores situados en lugares remotos.

Lo más significativo de las posibilidades interactivas que poseen las nuevas tecnologías de la información y comunicación, es que nos están permitiendo que el control de la comunicación, que durante bastante tiempo estaba situado en el emisor, se esté desplazando hacia el receptor, que determinará tanto el tiempo como la modalidad de uso. Al mismo tiempo éste podrá modificar su rol y adquirirá la función de transmisor de mensajes.

Esta interactividad, como apunta Bettetini (1995: 17), viene caracterizada por tres hechos básicos significativos: la pluridireccionalidad del deslizamiento de la información, el papel activo del usuario en la selección de las informaciones requeridas, y la comunicación en tiempo real. De acuerdo con este autor la interactividad podríamos definirla como un diálogo hombre-máquina, que haga posible la producción de objetos textuales nuevos, no completamente previsibles a priori.

Tecnologías interactivas que para Miller (1990) presentan una serie de ventajas: reducción del tiempo y el costo del aprendizaje, distribuyen la información de forma más consistente que la instrucción en vivo, intimidad en la interacción individual que se realiza con el material, dominio del propio aprendizaje, incremento de la retención, permiten poder explorar potencialmente los contenidos peligrosos sin riesgo, incremento de la motivación, facilita a la accesibilidad propiciando un aumento de la democratización de la educación, y permiten que los estudiantes puedan controlar su propio proceso de aprendizaje. Ahora bien, no debemos de perder de vista que la utilización de medios interactivos requiere una serie de peculiaridades que van desde un diseño específico, hasta la contemplación de un modelo de usuario, preocupado por la búsqueda de información o de nuevas asociaciones entre la misma.

Si hasta hace relativamente poco tiempo la influencia de las denominadas nuevas tecnologías de la información y comunicación se centraban fundamentalmente en los sectores militares, bancarios y de transferencia de comunicación de masas, en poco tiempo su impacto está alcanzado a todos los sectores de la sociedad, desde la 
enseñanza a la medicina, y desde el mundo del arte a la investigación de la información (Castells, 2006: 60).

\section{1. ¿Es la utilización de las nuevas tecnologías una cuestión de edad?}

Uno de los estereotipos de la sociedad actual, y nos atreveríamos a decir que también durante la historia de los medios de comunicación, es que el uso de las tecnologías se asocia por principio a la gente joven. Sin negar el hecho que toda nuevas tecnología es inicialmente utilizada en mayor grado por las personas más jóvenes, entre otros motivos porque el mundo en el cual se desenvuelven les ofrece más oportunidades para interaccionar con ellas; ello no debe entenderse como que están negadas para las personas de edades diferentes, sean éstos niños o mayores. No hay "a priori" ningún elemento que nos lleve a señalar, por ejemplo, que Internet está reservada a personas situadas dentro de una banda de edad.

Por el contrario cada vez nos encontramos con más experiencias apoyadas, o no, institucionalmente para la incorporación de estas tecnologías desde los primeros niveles de enseñanza. Y al mismo tiempo está aumentando de forma, pública y privada, la creación de entornos telemáticos para las personas mayores. Tal es el caso de empresas de diversos sectores como el bancario, que ponen a disposición de las personas mayores un entorno telemático para la interacción y el desarrollo de servicios.

Al mismo tiempo también se están potenciando bastante la creación de las comunidades virtuales que están formadas por un conjunto de personas conectadas a través de una red telemática, que les permite relacionarse entre sí para analizar temáticas específicas. Existen comunidades virtuales de todos los tipos, y entre ellas el fenómeno que viene a ser conocido como "panteras grises", es decir personas mayores conectadas a la red, se va progresivamente extendiendo en los países donde la tecnología de las redes se va haciendo rápida, y asequible.

\subsection{Las personas mayores y las nuevas tecnologías de la información y comunicación.}

Partiendo del principio que no tiene por qué haber usos diferenciados de las nuevas tecnologías en función de determinada banda de edad de los usuarios, sí podemos señalar los siguientes como los que más posibilidades ofrece: medio de interacción social, cultural, de ocio, ayuda, actividad laboral y formación.

Una de las posibilidades más significativas que las nuevas tecnologías juegan para las personas mayores, es la ayuda que les puedan prestar para superar uno de los mayores miedos que tienen estas personas: la soledad; tanto individual como en lo 
que respecta al aislamiento de sus familiares. De esta forma se aumenta la interacción en el ciberespacio, facilitándose con ello su autonomía personal y social.

Desde esta perspectiva, las nuevas tecnologías, y sobre todo Internet, pueden propiciar la creación de un entorno de comunicación, que facilite el desarrollo de las relaciones interpersonales y el contacto con su entorno, independientemente de la capacidad de movilidad del individuo o de la situación espaciotemporal donde se encuentre. Esta perspectiva sin lugar a dudas va a repercutir en abrir una nueva forma de comunicación para este colectivo de personas.

Estos entornos vienen favorecidos, por una parte por el hecho que la tecnología se está haciendo más amigable y por tanto más fácil de interaccionar con ella, y por otra, que los entornos que se le ofrecen son más interactivos y multimedias. Al mismo tiempo la extrañeza que hasta hace cierto tiempo despertaban las nuevas tecnologías se está perdiendo progresivamente, de forma que están adquiriendo mayor presencia en nuestra sociedad, como podemos observar por el aumento constante de diferentes anuncios sobre "portales" de Internet en televisión o porque cada vez va siendo más usual que las famosas "www" estén invadiendo las pantallas de televisión y los anuncios publicitarios. No cabe ya la menor duda que Internet se ha convertido en algo normal y usual dentro de nuestra cultura, ya empezamos a utilizar de forma natural el término "mundo en red".

Realizando un salto, y como redundancia de lo ya comentado de la asociación entre las nuevas tecnologías y las personas mayores, podemos observar en los últimos anuncios que sobre portales de Internet aparecen en la televisión, como aparecen imágenes de personas mayores, como queriendo llamar con ello la atención sobre este colectivo diana.

Todo esto está repercutiendo para que las asociaciones de personas mayores estén creando sitios web en Internet, desde donde ofrecer información a los usuarios de las diferentes actividades que realizan la asociación, información institucional, espacios para el intercambio de experiencia entre los sujetos, y zonas para la conversación e intercambio de experiencias.

En la evolución de las tecnologías de la información, sobre todo de las informáticas que son la base de las telecomunicaciones podríamos diferenciar dos grandes estadios, el primero, vendría caracterizado por la significación que tienen para la realización de las diferentes operaciones de forma lo más rápida y fiable posible; mientras que el segundo vendría avalado por su reconocimiento, influencia y utilización social. Dicho de otra forma, podríamos decir que las tecnologías que inicialmente se crearon como herramientas de cálculo y de escritura, han ido progresivamente transformándose en herramientas de comunicación, de forma que han pasado de ser herramientas para ampliar las capacidades del intelecto a ser herramientas para ampliar la presencia física y comunicativa del individuo. Desde estos últimos comentarios es desde donde podemos decir que las nuevas tecnologías, sobre todo en lo que respecta a la telemática, son antes que nada instrumentos que ponen en contacto a personas para comunicarse y relacionarse entre, superando las barreras espacio temporales. 


\section{OBJETIVOS Y METODOLOGÍA}

Entre los principales objetivos de la investigación se destacan los siguientes:

-Conocer las necesidades y la situación actual en la que se encuentran las personas mayores ante su relación de uso con las nuevas tecnologías en una zona rural2 ${ }^{2}$. -Averiguar qué herramientas y qué hábitos de uso /consumo tienen respecto a la utilización de Internet y las nuevas tecnologías.

-Saber cuál es el perfil dentro de una población mayor sobre el uso de Internet para mejorar su calidad de vida.

Para ello, se diseñó y realizó un cuestionario en el mes de septiembre de 2014 con el fin de conocer el perfil de usuario de Internet con una edad entre los 65 y 75 años. Se elaboró un cuestionario autoadministrado de cuatro preguntas cerradas y una abierta y se realizó previamente un pre test de dicho cuestionario a 5 personas que contaban con la edad sometida a estudio.

La finalidad era perfeccionarlo y detectar con suficiente antelación, posibles errores para su posterior difusión.

El cuestionario incluía en total 5 preguntas. La mayoría de ellas cerradas (respuesta de elección y excluyentes una de la otra). Hubo una pregunta abierta para conocer la opinión del encuestado (la número 5).

En general, la encuesta funcionó bien y no fue necesario introducir modificaciones importantes. Sólo se modificó el orden de algunas de las preguntas para que fueran más claras y enfocar mejor el tema de estudio al encuestado. Dado que la muestra era un grupo de personas entre 65 y 75 años, se diseñó un cuestionario corto y claro para la mejora de la comprensión de las preguntas; ya que era autoadministrado, es decir, el encuestador no se encontraba en presencia de la realización de dicho cuestionario.

\subsection{Estructura del Cuestionario}

En total como se ha dicho fueron 5 preguntas. Un cuestionario claro y corto para evitar al público de una edad más avanzada a contestar fácilmente. Inicialmente en el encabezado del mismo se explicaban los objetivos que se buscaban en el estudio y la Universidad que realizaba la investigación. Los cuestionarios eran totalmente anónimos.

Las preguntas se detallan a continuación:

2 Cabezón de Pisuerga es una localidad de Valladolid que se encuentra situada al norte a 12 kms de la ciudad. Tiene censadas un total de 4.200 personas, de las cuales 1410 personas cuentan con edades entre los 65 y 75 años. 312 personas entre 65 y 75 años tienen el carnet de socio y frecuentan la Casa del Mayor o Jubilado, por tanto se recogió una representación del 20\% del total (62 encuestados de las 312 personas en total). 
La primera pregunta, pretende saber cuánto tiempo se dedica al día a la conexión con Internet. La segunda pregunta intenta conocer el lugar donde se conecta el mayor. La tercera cuestión el dispositivo móvil o fijo de conexión.

Las últimas preguntas, 4 y 5 querían averiguar la justificación del uso de Internet. Concretamente la pregunta 4 sobre qué servicios e información se buscaba a través de la comunicación online. Esta pregunta era abierta totalmente.

La pregunta 5, aludía con opción de varias respuestas a la utilización actual de determinados servicios relacionados con las nuevas tecnologías como el uso de domótica, teleasistencia (cualquier servicio para facilitar la calidad de vida diaria y doméstica).

Finalmente para cerrar el cuestionario se agradeció la participación y se incluían preguntas de tipo sociodemográficas como:

Estudios realizados, ingresos actuales, edad y se consultaba también el estado conyugal de la persona (unidad familiar). Por último, se incluía una pregunta sobre los hobbies del mayor.

De esta forma, se podía relacionar el nivel de estudios y hobbies con el uso de Internet, al igual que la edad concreta de uso más regular del canal online.

Evidentemente, no hay que olvidar que nos centramos en un estudio que tiene lugar en una población rural. La investigación en un futuro podría seguir las líneas comparativas de uso de las nuevas tecnologías en una zona urbana frente a una zona rural por parte de los mayores.

\subsection{La Muestra}

La muestra se ha enfocado de forma seleccionada y restringida en una localidad de la provincia de Valladolid. Se contactó con la Asociación del Mayor en la población de Cabezón de Pisuerga para escoger una muestra de 62 personas mayores (edad comprendidas entre los 65 y 75 años) que acudían a las actividades programas por la Casa de Cultura del Ayuntamiento de Cabezón. De esta manera, en un lugar físico como es una dependencia de la Casa de Cultura, se informó en varias ocasiones del proyecto que se iba a llevar a cabo con las personas mayores.

\subsection{Distribución del Cuestionario}

El reparto y distribución del cuestionario tuvo una duración de un mes en total (septiembre de 2014).

Se solicitó permiso en el mes de julio al Ayuntamiento de Cabezón de Pisuerga para informar en tres ocasiones en la Casa del Mayor sobre el reparto del cuestionario. Por tanto, en la primera semana de septiembre se acudió varias veces para contar con una muestra asequible y representativa de las personas que acudían con regularidad y utilizaban las salas de informática en el pueblo (en la Casa del Mayor o Jubilado).

El cronograma fue el siguiente: 
1. Primero, se estableció contacto con el responsable de la Casa del Mayor, Ayuntamiento de Cabezón y Casa de Cultura del mismo pueblo. El contacto fue por teléfono y de forma presencial. Se realizó en julio de 2014.

2. Cuando se obtuvo el visto bueno por parte de los responsables, se estableció y fijó una fecha para entregarles de forma física y personal los cuestionarios en la casa del mayor.

3. Entre el 2 y el 5 de septiembre de 2014, se informó del proyecto a los mayores y se entregaron los cuestionarios autoadministrados.

4. Entre el 20 y 25 del mismo mes, se recogieron todos los cuestionarios y se preguntó por posibles incidencias surgidas.

5. Finalmente, se agradeció la colaboración por escrito al Ayuntamiento, Casa de Cultura y Casa del Mayor de Cabezón de Pisuerga, y se comenzó el análisis con los datos estadísticos obtenidos a partir de los cuestionarios.

Se recogieron 62 encuestas diferentes de las personas que habían acudido entre el 2 y 25 de septiembre a la Casa del Mayor o del Jubilado.

\section{RESULTADOS}

A continuación se muestran los resultados más importantes sobre los objetivos de la investigación:

Sobre los usos de Internet, la mayoría de ellos un 30\% (valor de 1) utilizan Internet para la búsqueda de información sanitaria y cultural. Existen valores similares de un $20 \%$ que fundamentalmente lo emplean para comunicarse y otro $20 \%$ para buscar entretenimiento. Finalmente, un 10\% lo utilizan para formación y trabajo y un $15 \%$ para contratar y pagar diversos servicios como viajes, cursos y obras culturales.

Figura 1. Usos de Internet

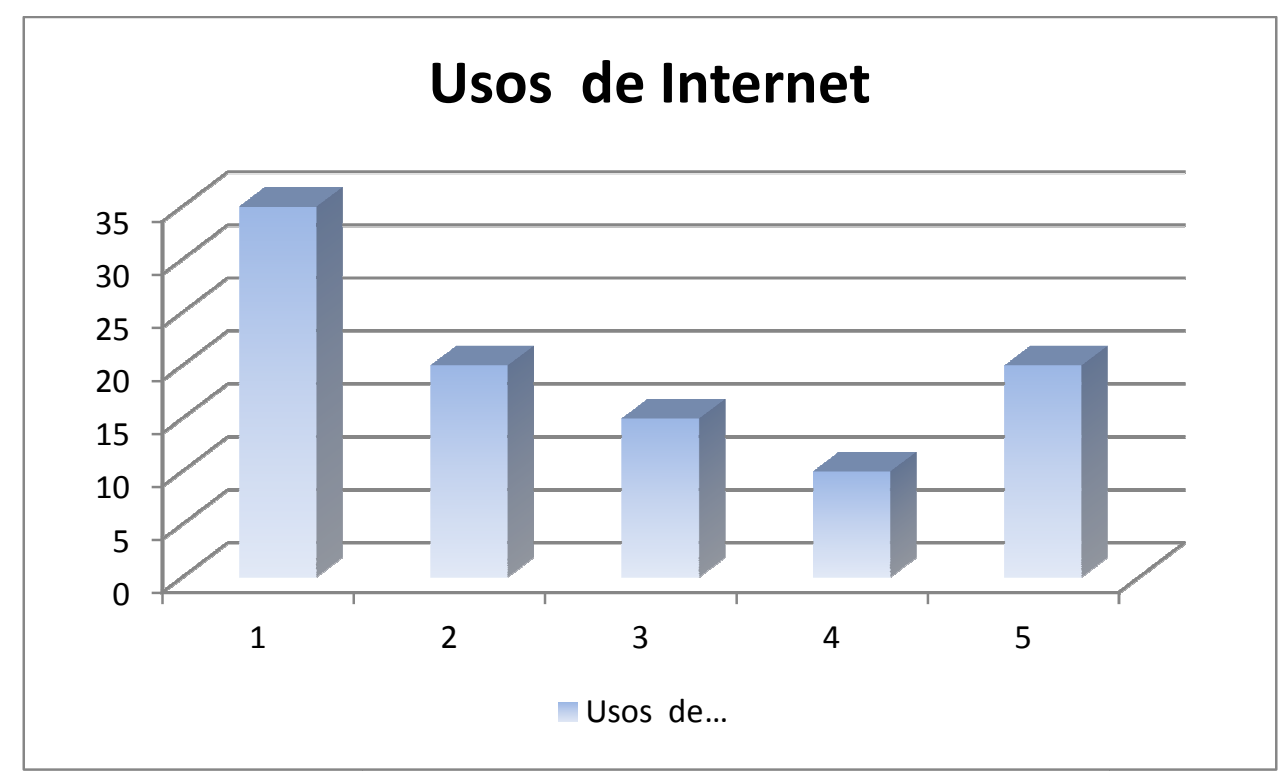


Respecto a las principales necesidades buscadas en este medio, se observa principalmente la necesidad de un $43 \%$ en la búsqueda de servicios relacionados con la mejora de la asistencia sanitaria a distancia, un $32 \%$ en comunicación con familiares y amistades, 15\% leer información y entretenimiento y un $10 \%$ en formación cultural.

Figura 2. Necesidades buscadas en Internet

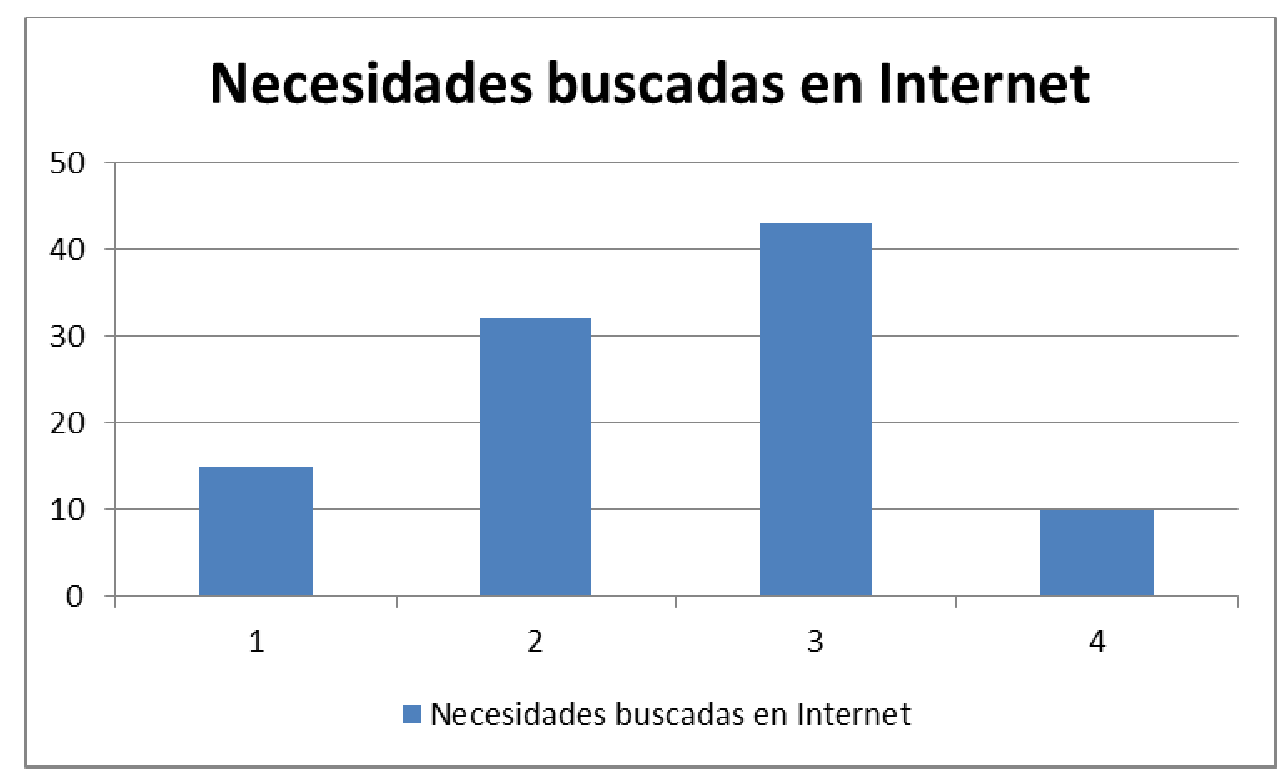

Fuente: elaboración propia

El perfil de usuario ${ }^{3}$ de este medio es un hombre entre 65 y 72 años, jubilado, con un poder adquisitivo medio-bajo (69\%), con estudios primarios $(72 \%)$ y secundarios (20\%). Tan sólo un $8 \%$ con estudios universitarios. Vive con su mujer (71\%) y un $29 \%$ de los hombres son viudos que vive solos o con hijos que residen en la misma población de Cabezón de Pisuerga. Sus hobbies principalmente son la pesca, el paseo y ver la televisión en casa.

${ }^{3}$ No hemos de olvidar que nos encontramos en una población rural. 
Figura 3. Género del encuestado

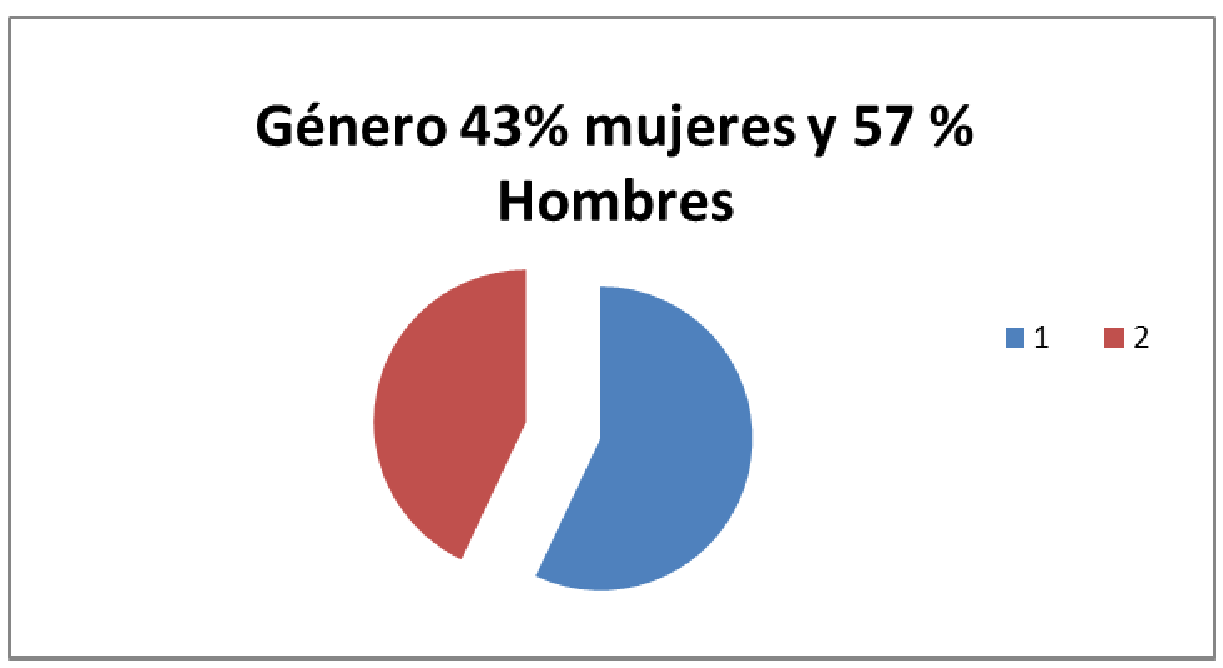

Fuente: elaboración propia

En cuanto al tiempo de exposición, es decir, tiempo que dedican a su conexión en la red, un $48 \%$ están una hora aproximadamente. Un $28 \%$ entre una y dos horas y, el $24 \%$ más de dos horas al día.

Se conectan principalmente a través de un dispositivo fijo (89\%) y el restante a través de un dispositivo móvil (sólo un 11\%). El lugar de conexión es desde la casa del jubilado un $78 \%$ y el otro $22 \%$, se conecta desde su hogar con un ordenador fijo prioritariamente.

Como se ha podido leer, uno de los aspectos más importantes y esenciales que vienen a potenciar las NTIC ${ }^{4}$, es el proceso comunicativo, configurándose éste en el elemento más importante en el transcurso del acto de enseñanza aprendizaje. Podemos definir la comunicación como el proceso que se desarrolla entre sujetos que disponen de algún tipo de convención y mediante su utilización intercambian algo, independientemente de la situación espacio-temporal de cada uno de ellos.

De acuerdo con Montero (2000), los mayores pueden aprender a través de programas y medios que han de tener en cuenta las características personales y las circunstancias que rodean este sector $y$, por supuesto, replantearse el concento educativo que ha dejado de lado dicha posibilidad. En este aspecto, hay que tener en cuenta que hablamos de una zona rural y la mayor preocupación de muchos de los encuestados

\footnotetext{
${ }^{4}$ Nuevas Tecnologías de la Información y la Comunicación.
} 
es la necesidad de información y de servicios de sanidad a través de Internet, es decir, ya sea asesoramiento y contacto médico que suele cubrir la asistencia a domicilio y la teleasistencia.

A la hora de analizar las posibles relaciones que se pueden dar entre las nuevas tecnologías y la formación, lo primero que tenemos que reconocer es que en la sociedad de la información se está potenciando el denominado aprendizaje a lo largo de toda la vida, superándose la idea de que éste se tiene que limitar a un período concreto de la vida, y a unas instituciones específicas, en las cuales adquiramos una serie de destrezas que nos permitan desenvolvernos a lo largo de toda nuestra vida social y laboral. En este ámbito al tratarse de una población no urbana, este uso y necesidad no se ve tan valorado como si se tratase de una zona urbana como fuera el ejemplo de Valladolid capital.

Es por ello que en determinadas poblaciones rurales se están llevando y desarrollando las Universidades de mayores, donde muchos de nuestros mayores están muy implicados en actividades socioculturales.

En este aspecto de formación no debemos de olvidar que las nuevas tecnologías han venido a romper las dos variables tradicionales en las que mayoritariamente se apoya nuestro sistema educativo: coincidencia espacio-temporal entre el profesor y el estudiante; potenciándose lo que se está viniendo a denominar como flexible y a distancia; es decir, aquella puede ser realizada utilizando las herramientas de comunicación sincrónica y asincrónica telemáticas. Por otra parte, la formación individualizada y flexible realizada fuera del contexto tradicional va adquiriendo mayor trascendencia como no había ocurrido anteriormente, entre otros motivos por asumir como principio operativo que cada persona tiene sus propias posibilidades y características de aprendizaje, unos ritmos y estilos de aprendizajes específicos y una disponibilidad horaria concreta.

Cabero y Barroso (1996), llegan a señalar que la formación en un futuro, independientemente del nivel educativo al que nos refiramos, vendrá determinada por diferentes características, como son: más individualizada, más flexible, basada en los recursos, accesible, a distancia, e interactiva.

Individualizada, ya que la educación tenderá progresivamente a responder a las necesidades concretas de los individuos, en lo que se está llamando como educación bajo demanda; es decir, respuestas educativas directas ante las solicitudes de formación realizadas expresamente por los estudiantes. Ello nos llevará a darle más significado a las características individuales de los sujetos y adaptar los procesos de formación a sus ritmos de aprendizaje y disponibilidad horaria. Este espíritu de formación nos llevará a la potenciación no de la enseñanza basada en el profesor, sino por el contrario en el estudiante, en torno a él girará el sistema educativo de la enseñanza.

Por otra parte nos encontramos que la formación tenderá a ser más flexible, ya que las ofertas y posibilidades educativas que se le ofrezcan a los ciudadanos no se 
limitarán únicamente a las instancias regladas y tradicionales del aprendizaje de su entorno cercano, sino que se abrirá un nuevo campo de posibilidades; de manera que podrá elegir cursos y propuestas de formación impartidas por instancias de formación diferentes del espacio en el cual él se encuentre. Esto permitirá que la formación de los individuos no se vea mediatizada por la calidad de los centros que tenga alrededor, sin olvidarnos de la posibilidad de solucionar uno de los problemas de las instituciones escolares, y es la lenta introducción con que los nuevos descubrimientos e ideas van llegando para su puesta a disposición de la comunidad de los que allí participan.

Al mismo tiempo esta flexibilidad debe de ser comprendida también desde la posibilidad de que se amplían los medios con los cuales puede interaccionar para el aprendizaje, teniendo a su disposición desde los tradicionales como la televisión y el libro de texto, hasta los más novedosos como los hipertextos o multimedias distribuidos.

\section{DISCUSIÓN Y CONCLUSIONES}

Cada vez se viene potenciando más la utilización de las nuevas tecnologías como instrumento de ayuda, asistencia sanitaria y de primeros auxilios a las personas mayores. En este sentido, son ya conocidos los aparatos de localización y solicitud de ayuda, tanto por vía telefónica como mediante videoconferencia por IP. Existiendo en cierta medida un servicio de vigilancia y atención sanitaria de la persona. No quedará mucho tiempo, para que una unidad de control reciba constantemente datos de determinadas constantes vitales de las personas, facilitándose de esta forma la teleasistencia de la persona para una medicina preventiva. Desde otra óptica, no debemos de olvidar que uno de los desarrollos del siglo XXI es el de la domótica, es decir, el sector de la informática que investiga el dotar de inteligencia artificial a los propios electrodomésticos para mejorar el uso de éstos por parte de determinados públicos como es el de la población más mayor. Ello permitirá que los frigoríficos previamente programados soliciten al supermercado los productos que nos hemos quedado sin ellos, dentro de una red de supermercados y analizando la relación calidad-precio, los electrodomésticos averiados se pondrán en contacto directo con los servicios técnicos de las casas comerciales; de modo que este tipo de situaciones problemáticas y complicadas para las personas mayores y que además viven solas, supone una ventaja considerable para mejorar la calidad de vida diaria.

Las nuevas tecnologías en general están propiciando el desarrollo de una nueva modalidad laboral, el denominado trabajo a domicilio o teletrabajo, que ocupará una parte importante del sector laboral en un futuro cercano (Cabero y Barroso, 1996). En líneas generales el teletrabajo lo que supone es la deslocalización del trabajador de la de la empresa, la realización de la actividad laboral en su propio domicilio y la utilización de medios telemáticos para las relaciones entre el trabajador y la empresa.

Las ventajas e inconvenientes que se han apuntado sobre la actividad laboral son diversas, y entre las primeras se encuentras: la posibilidad de ofrecer al trabajador la 
programación y organización del tiempo, evitar los costos y pérdidas de tiempo que supone el desplazamiento al centro de trabajo, organizar el tiempo de ocio en función de sus intereses, el ahorro que supone para la empresa ya que puede reducir el espacio y mobiliario necesario para llevar a cabo la actividad laboral, el facilitar a determinados colectivos como las mujeres y los sujetos con algún tipo de minusvalía su acercamiento al mundo laboral, el facilitar la presencia en la actividad laboral de las personas jubiladas, reducción para la empresa de los problemas del absentismo laboral, disminución de los tiempos no productivos, flexibilidad del mercado en cuanto a las relaciones laborales y la generación de nuevos puestos trabajos.

Por su parte, entre las segundas podemos indicar: aislamiento social del trabajador de la realidad y colectividad sociolaboral, la posibilidad de obligar al trabajador a estar disponible para la empresa durante las veinticuatro horas del día, costos adicionales para el trabajador que necesita disponer en su domicilio de una infraestructura tecnológica mínima y ampliar su zona particular de la vivienda para que pueda desarrollarse allí la actividad laboral, la inseguridad laboral para el trabajador ya que su vinculación con la empresa es menor, y las limitaciones que incorpora para la promoción dentro de la empresa.

No cabe la menor duda que además de las posibilidades que Internet ofrece para la comunicación y su campo de actuación en el terreno económico, otro de los grandes campos donde las nuevas tecnologías y especialmente Internet están ampliando su espectro es en el terreno de la cultura. Y en este sentido podemos empezar señalando que los bancos de datos de información y los sitios web en Internet se están ampliando notablemente. Internet está comenzando a acercarse a sus verdaderos retos de futuros y el ser una red de contenidos. El número de sitios web sobre los contenidos más diversos se está ampliando de forma inesperada, en la red posiblemente podamos encontrar de todo, aunque no debamos confundirlo con que lo encontremos, para ello tendremos que dominar no sólo la estructura de funcionamiento de la red, sino también poseer destrezas para la localización de información en la misma, habilidad que con la constante aparición de los portales, tanto temáticos como no, está haciendo que esta actividad sea de extraordinaria facilidad.

No obstante, la presente investigación pone de manifiesto que en las poblaciones rurales de la provincia de Valladolid como es el caso de Cabezón Pisuerga, los mayores tienen una preocupación mayor por la asistencia sanitaria y el contacto con este sector para el asesoramiento y búsqueda de información, es por ello que además de la formación, el entretenimiento y ocio, existe una importancia principal por la utilización de Internet con este fin, que es el de asegurar esta asistencia a las personas que viven solas y en las zonas rurales.

\section{REFERENCIAS}

Bettetini, G. (1995). Las nuevas tecnologías de la comunicación. Barcelona: Paidós. 
Cabero, J. (1994). Nuevas tecnologías, comunicación y educación. Comunicar, 3, 14-25.

Cabero, J. y Barroso, J. (1996). En el umbral del 2000. Formación ocupacional y nuevas tecnologías de la información: encuentros y desencuentro. Formación profesional ocupacional. Perspectivas de un futuro inmediato. Sevilla, GID-FETEUGT, 245-261.

Castells, J. (2006). La Sociedad Red. Madrid: Alianza Editorial.

Duby, A. (1991). Harnessing tv formats to educational tv, EMI, 28, 2, 70-74.

Gates, B. (1995). Camino al futuro. Barcelona: McGraw-Hill.

González, A.P. (1996). Las nuevas tecnologías en la formación ocupacional: retos y posibilidades. Revista de medio y educación, 2, 3-17.

Miller, R.L. (1990). Learning beneficts of interactive technologies. Videodisc Monitor, 8, 14-15.

Montero, I. (2000). El aprendizaje de los mayores ante los retos del nuevo milenio. Madrid: Dykinson.

Ramonet, I. (1997). Un mundo sin rumbo. Crisis de fin de siglo. Madrid: Temas de Debate.

Torres, J. (1994). Globalización e interdisciplinariedad: el currículum integrado. Madrid: Morata.

Turkle, Sh. (1997). La vida en la pantalla. Barcelona: Paidós.

\section{Mónica Matellanes-Lazo.}

Licenciada (2001) en Publicidad y RR.PP. por la Universidad Complutense de Madrid y Doctora (2009) por la Universidad de Valladolid en Comunicación del Enoturismo.

Ha trabajado en Comunicación y Marketing dentro del área vitivinícola en Bodegas Viña Pedrosa y Grupo Matarromera. En la actualidad es profesora agregada en Comunicación y Marketing en la Universidad Europea Miguel de Cervantes de Valladolid. Varias veces profesor Erasmus y congresista en Universidades extranjeras de Portugal (Lisboa, Ponte de Lima) y en Reino Unido (Aberystwyth). Autor de varios artículos de investigación en el área de Comunicación y la Web 2.0, últimas apariciones en Marketing y Ventas, Vivat Academia, Revista Deusto \& Harvard, Razón y Palabra. 